\title{
Características clínicas y patológicas en pacientes con cáncer de pulmón de células no pequeñas estadios I-IIIA
}

\author{
- Pedro Luis Ramos Guette, María Athenas Ramos Escalante, Diana Silva
}

Oncológica Oncocare (Bogotá, D.C.)

Introducción: el cáncer de pulmón es la primera causa de incidencia y mortalidad a nivel mundial. El propósito de este estudio es describir las características clínicas, patológicas, y la supervivencia de pacientes con cáncer de pulmón no metastásico estadios I a IIIA.

Materiales y métodos: se trata de un estudio observacional descriptivo retrospectivo realizado durante el período del $1^{\circ}$ de mayo de 2003 al 30 de febrero de 2015. Se incluyeron 38 pacientes mayores de 18 años con diagnóstico de cáncer de pulmón estadios I a IIIA. Se hicieron análisis descriptivos usando medidas de tendencia central, ubicación y dispersión para las variables continuas, y frecuencias absolutas y relativas para las variables categóricas. Se estimó la supervivencia por el método de Kaplan-Meier.

Resultados: se identificó un total de 38 pacientes en el estudio con edad promedio de 65,8 años. El $65,7 \%$ son masculinos. El $55 \%$ de los pacientes se encontraban en estadios I y el estado funcional era ECOG 0-1 en un $89 \%$. El $67 \%$ de los pacientes tenía adenocarcinoma y el $71 \%$ recibió quimioterapia adyuvante. A la mayor parte de los pacientes se le administró platinos más vinorelbina o placlitaxel (55\%). Se presentó una frecuencia del $26,3 \%$ de recaídas y un $31 \%$ de muertes. La mediana de seguimiento fue de 68 meses.

Conclusiones: la mayoría de los pacientes con cáncer de pulmón presenta una histología de adenocarcinoma y estadio I con buen estado funcional, con una frecuencia de recaída y muerte de acuerdo con la literatura. 